\title{
Bei Rückenschmerzen reicht zunächst ein NSAR
}

\author{
Akute Rückenschmerzen werden in der Regel mit mehreren Medikamenten gleichzeitig \\ bekämpft. Eine neue klinische Studie legt nahe, sich zunächst auf ein NSAR zu beschränken.
}

— Nach den Leitlinienempfehlungen sollten akute Rückenschmerzen über einen begrenzten Zeitraum mit nicht steroidalen Antirheumatika (NSAR) und/oder Muskelrelaxanzien behandelt werden. Teilweise werden auch Opioide empfohlen. Ein guter Vergleich zwischen den Kombinationen NSAR plus Muskelrelaxanzien und Opioide plus Paracetamol fehlte bisher.

In die Studie wurden 323 Patienten im mittleren Alter von 39 Jahren aufgenommen, die sich mit akuten Rückenschmerzen in einer Notfallaufnahme vorstellten. Patienten mit radikulären Schmerzen wurden ausgeschlossen. Die Teilnehmer erreichten bei Studieneinschluss einen Wert von mindestens 5 Punkten im Roland Morris Disability Questionnaire (RMDQ), das auf einer Skala von 0-24 den Schmerz und die
Einschränkung bei Rückenschmerzen misst. Alle Patienten erhielten als Basistherapie zweimal täglich ein NSAR, nämlich 500 mg Naproxen. Zusätzlich bekamen sie täglich entweder Placebo, ein Muskelrelaxans oder Oxycodon in Kombination mit Paracetamol. Der primäre Endpunkt war die Schmerzreduktion im RMDQ nach einer Woche.

Die Patienten litten zum Zeitpunkt der Randomisierung seit 48 Stunden unter Rückenschmerzen. Der Median des RMDQ-Scores betrug 20 Punkte. Etwa die Hälfte der Patienten hatte bereits früher unter Rückenschmerzen gelitten. Mit Placebo besserten sich die Rückenschmerzen um 9,8 Punkte, mit dem Muskelrelaxans um 10,1 Punkte und mit dem Opioid plus Paracetamol um 11,1 Punkte. Die Gruppenunterschiede waren statistisch nicht signifikant.
- Friedman BW, Dym AA, Davitt Met al. Naproxen with cyclobenzaprine, oxycodone/acetaminophen, or placebo for treating acute low back pain: A randomized clinical trial. JAMA 2015;314:1572-80

\section{KOMMENTAR}

Die Ergebnisse dieser Studie haben Konsequenzen für die Versorgung von Patienten mit akuten Rückenschmerzen. Offenbar reicht es zunächst aus, ein ausreichend dosiertes NSAR zu geben. Diese Monotherapie kann man eine Woche lang versuchen. Die zusätzliche Gabe von Muskelrelaxanzien oder einer Kombination Opioid/Paracetamol erbringt offenbar keinen zusätzlichen Nutzen im Hinblick auf die Reduktion der Rückenschmerzen innerhalb dieser Zeitspanne. Patienten mit radikulären Schmerzen waren allerdings von der Teilnahme an dieser Studie ausgeschlossen. Für sie gelten die Ergebnisse dementsprechend nicht.

Prof. Dr. med. H.-C. Diener

\section{Im EKG zeigt sich kardialer Rechtsextremismus}

Ein 61-jähriger Mann mit Nikotinabusus und einer Familienanamnese von koronarer Herzkrankheit erwachte mit einem linksthorakalen Druckgefühl, Schwindel und Schweißausbrüchen. Die Herzfrequenz betrug 85/min, der Blutdruck nur 90/60 mmHg. Das EKG bei Aufnahme zeigte einen Sinusrhythmus mit ST-Erhebungen in den Ableitungen II, III, aVF sowie V1 (Abb. A).



Wegen der ST-Hebung in V1 vermutete man einen rechtsventrikulären Myokardinfarkt und zeichnete auch rechtsthorakale Ableitungen auf. In diesen erkannte man einen AV-Block mit 2:1-Überleitung und ausgeprägte STHebungen in den Ableitungen rV3 bis rV6, womit die Infarzierung der inferioren Ventrikelanteile mit Einbeziehung des rechten Ventrikels bewiesen war (Abb. B).

Nach Infusionstherapie und temporärer Schrittmacherbehandlung ergab eine Koronarangiografie einen vollständigen Verschluss der rechten Koronararterie (Abb. C, Pfeil). Nach einer perkutanen transluminalen Koronarangioplastie (PTCA) stellten sich die rechte Koronararterie (Abb. D, schwarzer Pfeil) und deren rechtsventrikulärer hinterer Ast (weißer Pfeil) wieder als gut durchblutet dar. Danach verbesserte sich die hämodynamische Situation des Patienten. Eine Kontroll-Echokardiografie ergab eine normale linksventrikuläre systolische Funktion und nur eine leichte rechtsventrikuläre Dysfunktion.

Prof. Dr. med. H. S. FüeßI

- Namana V, Balasubramanian R(vnamana@maimonidesmed.org). Right ventricular infarction. N Engl J Med. 2016;374:872 